\title{
FBW7 increases the chemosensitivity of pancreatic cancer cells to gemcitabine through upregulation of ENT1
}

\author{
QIANGSHENG HU ${ }^{1-3^{*}}$, YI QIN ${ }^{1-3^{*}}$, BO ZHANG $^{1-3^{*}}$, CHEN LIANG $^{1-3}$, SHUNRONG JI $^{1-3}$, \\ SI SHI ${ }^{1-3}$, WENYAN XU ${ }^{1-3}$, JINFENG XIANG ${ }^{1-3}$, DINGKONG LIANG ${ }^{1-3}$, \\ QUANXING NI ${ }^{1-3}$, XIANJUN YU ${ }^{1-3}$ and JIN XU ${ }^{1-3}$ \\ ${ }^{1}$ Department of Pancreatic Surgery, Fudan University Shanghai Cancer Center; \\ ${ }^{2}$ Department of Oncology, Shanghai Medical College, Fudan University; \\ ${ }^{3}$ Pancreatic Cancer Institute, Fudan University, Shanghai 200032, P.R. China
}

Received January 11, 2017; Accepted May 24, 2017

DOI: $10.3892 /$ or.2017.5856

\begin{abstract}
F-box and WD repeat domain-containing 7 (FBW7) has been characterized as a tumor suppressor, and its mutation or decreased expression has been observed in many types of human cancers. Our recent studies have uncovered that in pancreatic cancer, the KRAS mutation decreased FBW7 expression through phosphorylation and subsequent ubiquitination. Moreover, FBW7 inhibited aerobic glycolysis in pancreatic cancer via induction of thioredoxin-interacting protein (TXNIP), a mitochondrial localized tumor suppressor. The roles of FBW7 in anti-apoptosis and drug resistance via proteosomal degradation of myeloid cell leukemia-1 (MCL-1), which is an anti-apoptotic factor have been reported. However, the role of FBW7 in the chemotherapeutic resistance of pancreatic cancer to gemcitabine has seldom been reported. In the present study, we demonstrated that overexpression of FBW7 in pancreatic cancer cells rendered increased sensitivity to gemcitabine. Mechanistically, FBW7 promoted gemcitabine sensitivity via upregulation of equilibrative nucleoside transporter 1 (ENT1) at the protein level rather than the transcriptional level. In depth analysis demonstrated that the ENT1 protein level could be increased by lysosome inhibition. Taken together, our results demonstrated that FBW7 could be a target for improving the therapeutic efficacy of gemcitabine by induction of ENT1.
\end{abstract}

Correspondence to: Dr Jin Xu or Dr Xianjun Yu, Department of Pancreatic Surgery, Fudan University Shanghai Cancer Center, Shanghai 200032, P.R. China

E-mail: xujin@fudanpci.org

E-mail: yuxianjun@fudanpci.org

${ }^{*}$ Contributed equally

Key words: pancreatic cancer, gemcitabine, chemosensitivity, FBW7, ENT1

\section{Introduction}

Pancreatic cancer is one of the most aggressive and lethal malignant diseases worldwide. Due to the lack of early detection techniques, the disease is usually diagnosed at an advanced stage. Moreover, the highly aggressive and invasive nature of this disease makes surgical resection difficult since at diagnosis, it usually has metastasized to nearby and distant organs. Thus, only a small portion of patients are indicated for surgery, and even in this case, the 5-year survival rate remains disappointedly steadily at $\sim 7 \%(1,2)$. Chemotherapy is an important alternative for the systematic treatment of pancreatic cancer (3). However, conventional radiotherapy and chemotherapies have limited efficacy in improving the overall survival of patients diagnosed with pancreatic cancer, since this disease is highly resistant to almost all chemotherapeutic agents and traditional radiotherapies (4). Gemcitabine (2',2'-difluorodeoxycytidine) is the standard first-line anticancer agent for pancreatic cancer (5). Although gemcitabine shows significant benefits in patients, the response rate and prognosis remain disappointing and it shows a modest benefit in terms of progression-free, disease-free and overall survival (6). Therefore, any strategy that enhances the sensitivity of pancreatic cancer to gemcitabine may improve the prognosis of this fatal disease.

F-box and WD repeat domain-containing 7 (FBW7) is the substrate recognition subunit of an Skp1-Cullin1-F-box (SCF) ubiquitin ligase complex. FBW7 is deregulated in $\sim 10 \%$ of all human cancers, usually by point mutations or decreased expression by epigenetic modifications (7). FBW7 possesses tumor-suppressive roles by targeting a series of onco-proteins for degradation, including c-Myc, cyclin E, Jun, Notch intracellular domain (NICD), hypoxia inducible factor- $1 \alpha$ (HIF-1 $\alpha$ ), Krüppel-like factor 5 (KLF5) and MCL-1 (8). Through mutations or epigenetic silencing, decreased FBW7 expression drives proliferation, attenuates apoptosis, induces genomic instability and maintains stem-cell properties, which ultimately promotes tumorigenesis $(9,10)$. Our previous studies demonstrated that in pancreatic cancer, the protein level of FBW7 was tightly controlled by oncogenic Kras/ERK signaling. Decreased FBW7 caused an increase in oncoprotein 
c-Myc, which promoted pancreatic cancer proliferation and progression (11). Moreover, decreased FBW7 expression was found to render metabolic advantages to pancreatic cancer cells by inducing aerobic glycolysis through downregulation of thioredoxin interacting protein (TXNIP) in a c-Mycdependent manner (12). Furthermore, we demonstrated that decreased FBW7 expression induced cancer antigen 125 (CA125) or Muc16 production in pancreatic cancer cells, which promoted the metastatic capacity of pancreatic cancer cells (13). However, the contribution of FBW7 to pancreatic cancer chemotherapy and the underlying mechanisms have seldom been discussed.

Key determinants of gemcitabine cytotoxicity include activities of the equilibrative nucleoside transporter 1 (ENT1), deoxycytidine kinase (dCK) and ribonucleotide reductase subunit 1 (RRM1) (14). Gemcitabine is a highly hydrophilic chemical, and passive diffusion through the hydrophobic membrane is slow. Thus, it requires transporters to facilitate its intake. ENT1 is the membrane transporter protein that helps efficient permeation of gemcitabine into cells $(15,16)$. Gemcitabine is a prodrug, and it must be phosphorylated by $\mathrm{dCK}$ as the rate limiting step for its cellular anabolism. dCK catalyzes gemcitabine monophosphate to its active metabolites, gemcitabine diphosphate and gemcitabine triphosphate. One mechanism of gemcitabine cytotoxicity is blocking de novo DNA synthesis through the inhibition of ribonucleotide reductase, thereby blocking production of the deoxyribonucleotide precursors needed for DNA synthesis. RRM1 and RRM2 are components that can be inactivated by difluorodeoxycytidine- 5 phosphate. The triphosphorylated form of gemcitabine can be incorporated into DNA and leads to chain termination during DNA synthesis, promoting the apoptosis of pancreatic cancer cells (17). Previous studies using large multicenter cohorts of patients with resected pancreatic cancer suggest that ENT, dCK and RRM1 levels predict the efficacy of gemcitabine and patient prognosis (14).

In the present study, we analyzed the contribution of FBW7 to gemcitabine resistance in pancreatic cancer, and indicated that FBW7 increased the sensitivity to gemcitabine. We demonstrated that anti-apoptotic player MCL-1 was not influenced by FBW7 in pancreatic cancer cells. Thus, we examined the effect of FBW7 on ENT, dCK and RRM1. We demonstrated that among these determinants of gemcitabine efficacy, FBW7 regulated the ENT1 protein level. Moreover, membrane-bound ENT1 was increased in the FBW7overexpressing cells. Finally, we demonstrated that the ENT1 level was influenced by lysosome inhibition instead of proteosomal inhibition, indicating a novel regulatory mechanism in ENT1 regulation. Collectively, our results provide novel targets for improving gemcitabine resistance in pancreatic cancer.

\section{Materials and methods}

Cell culture. Human pancreatic cancer cell lines PANC-1 and Mia PaCa-2 were obtained from the Shanghai Cell Bank (Shanghai, China). PANC-1 cells were cultured in Dulbecco's modified Eagle's medium (DMEM) supplemented with 10\% fetal bovine serum (FBS), $100 \mathrm{U} / \mathrm{ml}$ penicillin and $0.1 \mathrm{mg} / \mathrm{ml}$ streptomycin. As for Mia PaCa-2 cells, an additional 2.5\%
Table I. Primer sequences used in quantitative real-time PCR.

\begin{tabular}{lll}
\hline ENT1 & F & 5'-CTCCAACTCTCAGCCCACCAATGA-3' \\
& R & 5'-GAAGTAACGTTCCCAGGTGCTGC-3' \\
dCK & F & 5'-CAAGACTGGCATGACTGGATGAA-3' \\
& R & 5'-GGCACCTCTTGAAGATAATCGAAG-3' \\
\multirow{2}{*}{ RRM1 } & F & 5'-TGGAGTACACCAGCAAAGATGAGG-3' \\
& R & 5'-GGCGATGGCGTTTATTTGATAGGC-3' \\
\multirow{3}{*}{-actin } & F & 5'-CTACGTCGCCCTGGACTTCGAGC-3' \\
& R & 5'-GATGGAGCCGCCGATCCACACGG-3' \\
\hline
\end{tabular}

F, forward; R, reverse; ENT1, equilibrative nucleoside transporter 1; dCK, deoxycytidine kinase; RRM1, ibonucleotide reductase subunit 1 .

horse serum was used for its culture. The cells were maintained in a humidified incubator at $37^{\circ} \mathrm{C}$ with $5 \% \mathrm{CO}_{2}$.

Establishment of cell lines stably expressing FBW7. PANC-1 and Mia PaCa-2 cell lines that stably expressed FBW7 were established by lentiviral-mediated transfection. pCDHCMV-MCS-EF1-Puro (System Biosciences, Palo Alto, CA, USA) was used for generation of the lentiviral-expressing constructs. Lentiviral particles were obtained by co-transfection of lentiviral constructs of FBW7 with psPAX2 and pMD2.G vectors into 293T cells in a ratio of 4:3:1. Stable cells lines were obtained by infection and subsequent selection by puromycin.

Cell viability assay. Cell viability was measured using the Cell Counting Kit-8 (Dojindo, Tokyo, Japan). Briefly, $200 \mu \mathrm{l}$ medium containing cells (3,000/well) was seeded into 96-well plates. After culturing for the indicated times, CCK-8 solution was added to each well at $37^{\circ} \mathrm{C}$. After $2 \mathrm{~h}$, the optical density (OD) values of each well were measured using a microplate reader at a wavelength of $450 \mathrm{~nm}$.

Cell apoptosis analysis. Cell apoptosis was assessed using flow cytometry. For the cell apoptosis assay, PANC-1 and Mia PaCa-2 cells stably transfected with FBW7 were incubated in the absence or presence of gemcitabine for 24 or $48 \mathrm{~h}$. The percentage of apoptotic cells was analyzed by staining with fluorescein isothiocyanate-conjugated Annexin V and propidium iodide (Invitrogen, Carlsbad, CA, USA), followed by flow cytometric testing.

Quantitative real-time PCR. Total RNA was extracted using TRIzol reagent (Invitrogen). Takara PrimeScript RT reagent kit was used for reverse transcription to obtain cDNA (Takara, Tokyo, Japan). The expression status of the candidate genes and $\beta$-actin was determined by quantitative real-time PCR using an ABI 7900HT Real-Time PCR System (Applied Biosystems, Foster City, CA, USA). Primer sequences are listed in Table I.

Western blotting. Cells were lysed in RIPA buffer $(150 \mathrm{mM}$ $\mathrm{NaCl}, 1 \% \mathrm{NP}-40,50 \mathrm{mM}$ Tris/HCl, $\mathrm{pH} 8.0$ and $10 \%$ glycerol) containing protease and phosphatase inhibitors purchased from Selleck (Houston, TX, USA). Cell debris 
was removed by centrifugation at $12,000 \mathrm{rpm}$ for $20 \mathrm{~min}$ at $4^{\circ} \mathrm{C}$. Thermo Pierce ${ }^{\circledR}$ BCA Protein Assay kit was used to test the protein concentrations. Equal amounts of protein lysates were subjected to $10 \%$ SDS-PAGE and then transferred to polyvinylidene difluoride (PVDF) membranes. The FBW7 antibody was purchased from Bethyl Laboratories (Montgomery, TX, USA). Antibodies against ENT1, RRM1 and $\beta$-actin were obtained from Proteintech (Chicago, IL, USA). The dCK antibody was obtained from Abcam (Cambridge, MA, USA).

Membrane protein extraction. In order to detect the distribution of proteins on the cell membrane, Mem-PER ${ }^{\mathrm{TM}}$ Plus Membrane Protein Extraction kit (Thermo Fisher Scientific, Inc., Waltham, MA, USA) was used for the extraction of membrane proteins. APT1A1 and TFR antibodies that were purchased from Proteintech were used as loading controls.

Immunohistochemistry. Paraffin-embedded tissue slides were deparaffinized in xylene, rehydrated through graded alcohol solutions, blocked in methanol containing 3\% hydrogen peroxide, and then incubated in the FBW7 and ENT1 antibodies. Following rinsing with phosphate-buffered saline (PBS) solution, the slides were incubated with secondary antibodies and peroxidase reagent at room temperature. Finally, the slides were incubated with 3,3'-diaminobenzidine solution at room temperature for $10 \mathrm{~min}$ and counterstained with hematoxylin. The use of human PDAC tissue specimens was evaluated and approved by the Ethics Committee of Fudan University Shanghai Cancer Center, and written informed consent was obtained from all participants.

Immunofluorescence. To observe the cellular distribution and endogenous levels of the indicated proteins, immunofluorescence assay was performed. In brief, the cells were fixed with $4 \%$ paraformaldehyde at room temperature. Then, $0.5 \%$ Triton X-100/PBS solution was used to permeabilize the cells. After washing the slides with phosphate-buffered saline with Tween-20 (PBST)/bovine serum albumin (BSA), the slides were blocked with PBST/BSA for $1 \mathrm{~h}$ at room temperature. The cells on the slides were stained with primary antibodies followed by PBST washing for five times, and then secondary antibodies were applied. Vectashield ${ }^{\circledR}$ Mounting Medium with 4',6-diamidino-2-phenylindole (DAPI) was used to label DNA and prevent slow fading of the fluorescence. A laser scanning confocal microscope (Leica Microsystems, Wetzlar, Germany) was used to detect fluorescence.

\section{Results}

FBW7 increases sensitivity to gemcitabine. To assess the role of FBW7 in the efficacy of gemcitabine on pancreatic cancer cells, we overexpressed FLAG-tagged FBW7 in PANC-1 and Mia PaCa-2-2 cells, and the effect of overexpression was validated by immunoblotting with FLAG antibody (Fig. 1A). We next studied the chemosensitivity to gemcitabine in the FBW7-overexpressing PANC-1 and Mia PaCa-2 cells. Empty vector (EV)-transfected and FBW7-overexpressing (FBW7) pancreatic cancer cells were treated with the indicated concentrations of gemcitabine for $72 \mathrm{~h}$. Significantly lower $\mathrm{IC}_{50}$ values were observed in the FBW7-overexpressing PANC-1 and Mia PaCa-2 groups (Fig. 1B). Next, we performed cell apoptosis assay, and our results demonstrated that introduction of FBW7 increased the apoptosis of PANC-1 and Mia $\mathrm{PaCa}-2$ cells upon gemcitabine treatment, compared to the relative control groups (Fig. 1C and D). Moreover, we observed an increase in the cleaved PARP1 level in the FBW7overexpressing cells treated with gemcitabine, indicating an increase in apoptosis (Fig. 1E). Taken together, the results indicated that FBW7 overexpression increased sensitivity to gemcitabine in pancreatic cancer cells.

$F B W 7$ regulates the ENT1 protein level. To ascertain the underlying molecular mechanism accounting for the effect of FBW7 on gemcitabine sensitivity, we first examined the protein level of MCL1, an anti-apoptotic protein that accounts for drug resistance in many types of cancers. MCL-1 has been reported to be an FBW7 substrate, but we detected no change in the MCL-1 protein level in the FBW7-overexpressing PANC-1 and Mia PaCa- 2 cells, indicating that there may be other molecular mechanisms (Fig. 2A). ENT1, dCK and RRM1 have been reported to be key factors indicating gemcitabine efficacy in pancreatic cancer (Fig. 2B). Thus, we detected the mRNA levels of these genes in the FBW7-overexpressing PANC-1 and Mia PaCa-2 cells, but we detected no obvious changes in the mRNA levels (Fig. 2C). Next, we examined the protein level of these factors, and observed that FBW7 overexpression significantly increased ENT1 at the protein level, but had slight impact on the RRM1 protein level (Fig. 2D). Moreover, using two separate antibodies produced by different manufacturers, we detected no dCK level in the PANC-1 and Mia PaCa-2 cells. These results indicate that the regulation of gemcitabine resistance by FBW7 may due to the impact of the increase in ENT1 protein level.

FBW7 regulates ENT1 membrane distribution. ENT1 membrane localization is responsible for gemcitabine efficient uptake. Thus, we examined the membrane localization of ENT1 in FBW7-overexpressing PANC-1 and Mia PaCa-2 cells; loading controls were used by examining the protein levels of TFR or ATP1A1. Our results revealed that ATP1A1 was markedly altered, while no change in TFR was observed. Thus, we took TFR as a membrane loading control. In addition, our results indicated that overexpression of FBW7 increased the membrane distribution of ENT1 (Fig. 3A). Furthermore, our results demonstrated that overexpression of FBW7 increased the total level of ENT1 and membranebound ENT1 as reflected by the immunofluorescence assay (Fig. 3B). To further confirm the regulatory role of FBW7 on ENT1, we performed IHC staining in patients with PDAC. As observed, the ENT1 level was significantly higher in patients that displayed higher FBW7 expression, reflecting a positive correlation between FBW7 and ENT1 in PDAC patients (Fig. 3C). Factors that are involved in protein levels include proteasome-mediated degradation and lysosomal degradation. Thus, we treated PANC-1 and Mia PaCa- 2 cells with proteasome inhibitor MG132, but detected no obvious change in ENT1 levels in the PANC-1 and Mia PaCa-2 cells (Fig. 3D). Then, we treated cells with one lysosome inhibitor chloroquine (CQ), and observed a significant 
A

PANC-1

EV FBW7 $7^{\text {FLAG }}$

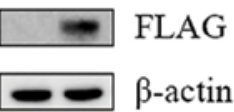

C
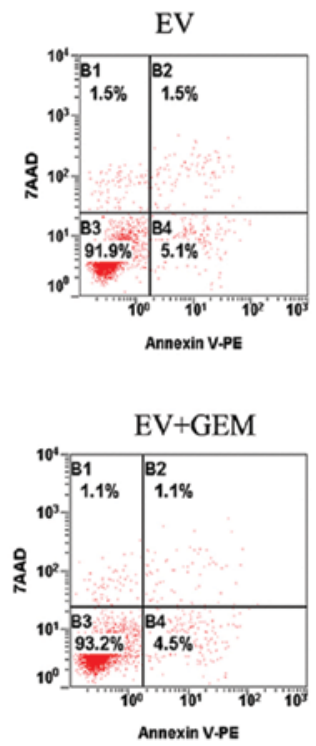

D

PANC-1

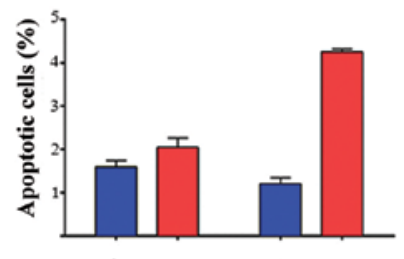

$\mathrm{EV}+-+-$

FBW7 - + -+
Mia PaCa-2

EV FBW7 $7^{\text {FAG }}$

FLAG

$\square$ B-actin

PANC-1

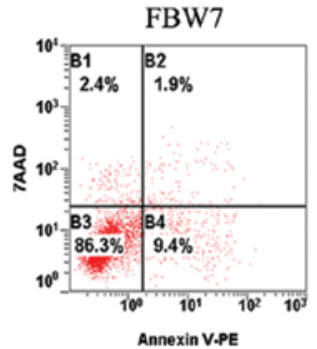

FBW7+GEM

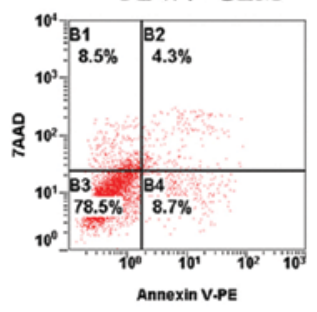

Mia PaCa-2

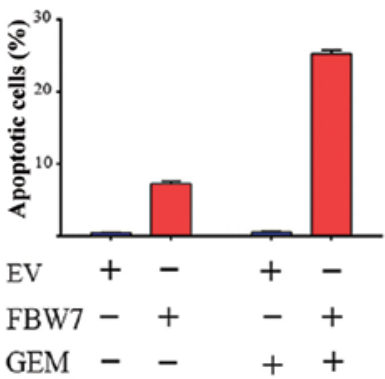

$\mathrm{B}$

PANC-1

Mia PaCa-2
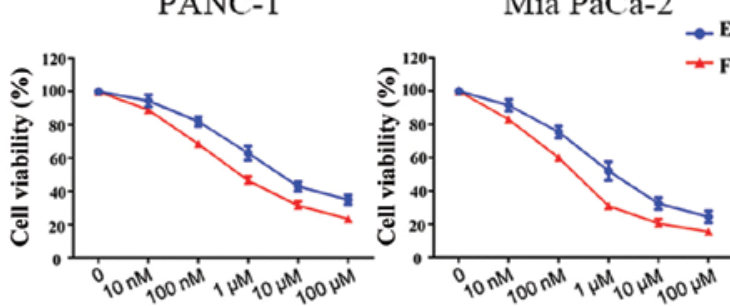

Mia PaCa-2
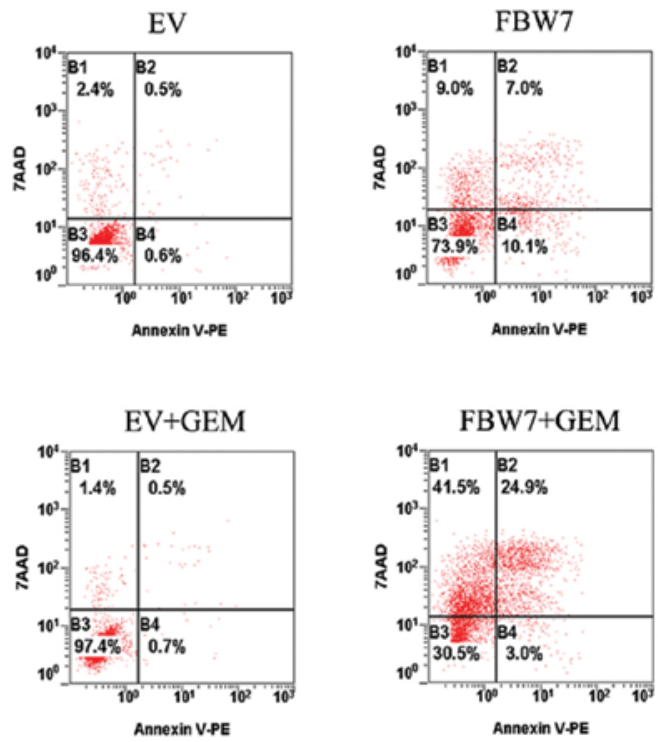

FBW7+GEM

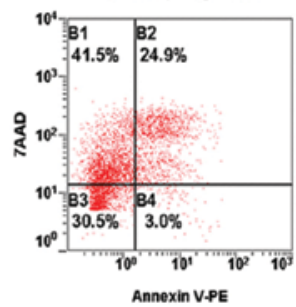

E

PANC-1

Figure 1. FBW7 increases the sensitivity of pancreatic cancer cells to gemcitabine. (A) FBW7 was introduced into PANC-1 and Mia PaCa-2 cells by lentiviralmediated transfection, and the overexpression efficacy was assessed by detection with FLAG antibodies. (B) PANC-1 and Mia PaCa- 2 cells were treated with increasing concentrations of gemcitabine (GEM) (0.01-100 $\mu \mathrm{M})$ for $72 \mathrm{~h}$. The cell viability ratios were measured by CCK- 8 assays. In PANC-1 cells, FBW7 decreased the $\mathrm{IC}_{50}$ value from 10 to $2 \mu \mathrm{M}$, and in Mia PaCa- 2 cells, the $\mathrm{IC}_{50}$ value changed from 2 to $0.5 \mu \mathrm{M}$. (C and D) Gemcitabine increased the cell apoptosis in the FBW7-overexpressing PANC-1 and Mia PaCa-2 cells as reflected by analysis of apoptosis. (E) The protein level of cleaved PARP1 was significantly increased in the gemcitabine-treated, FBW7-overexpressing PANC-1 and Mia PaCa-2 cells, indicating that FBW7 renders pancreatic cancer cells sensitive to gemcitabine. GEM, gemcitabine.

increase in the ENT1 protein level. These results suggested a novel lysosome-mediated regulation of ENT1 in pancreatic cancer cells (Fig. 3E). Further experiments demonstrated that combination of CQ application increased the sensitivity to gemcitabine in the PANC-1 and Mia PaCa-2 cells (Fig. 3F). Moreover, our results demonstrated that treatment with the combination of CQ and gemcitabine increased the level of cleaved PARP1, further supporting the hypothesis that lysosome-induced ENT1 degradation may participate in the acquisition of gemcitabine resistance (Fig. 3G).
FBW7 upstream regulator ERK regulates ENT1 abundance. Our previous studies (11) demonstrated that ERK phosphorylates and destabilizes FBW7 in pancreatic cancer cells. To ascertain whether ERK regulates ENT1 abundance in pancreatic cancer cells, we first treated pancreatic cancer cells with MEK inhibitor UO126, and our results indicated that ERK inhibition upregulated the protein levels of ENT1 (Fig. 4A). Furthermore, immunofluorescence results also demonstrated that UO126 treatment elevated ENT1 levels (Fig. 4B). Next, we performed membrane protein purification, and observed 
A

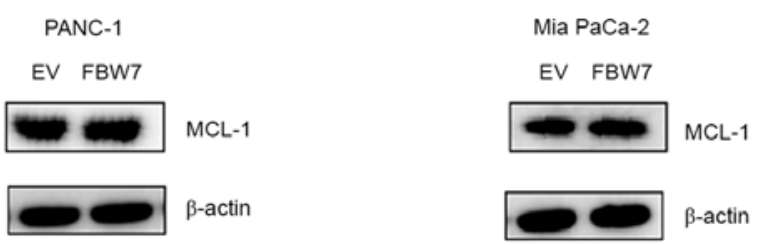

B

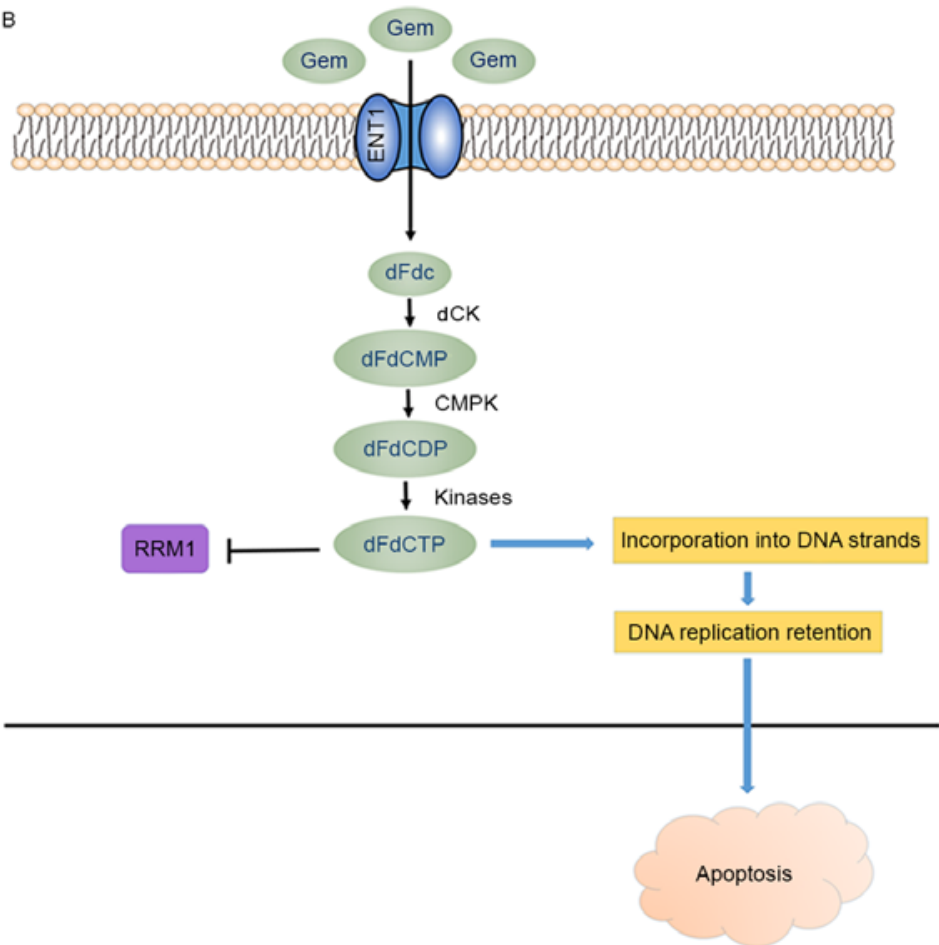

C
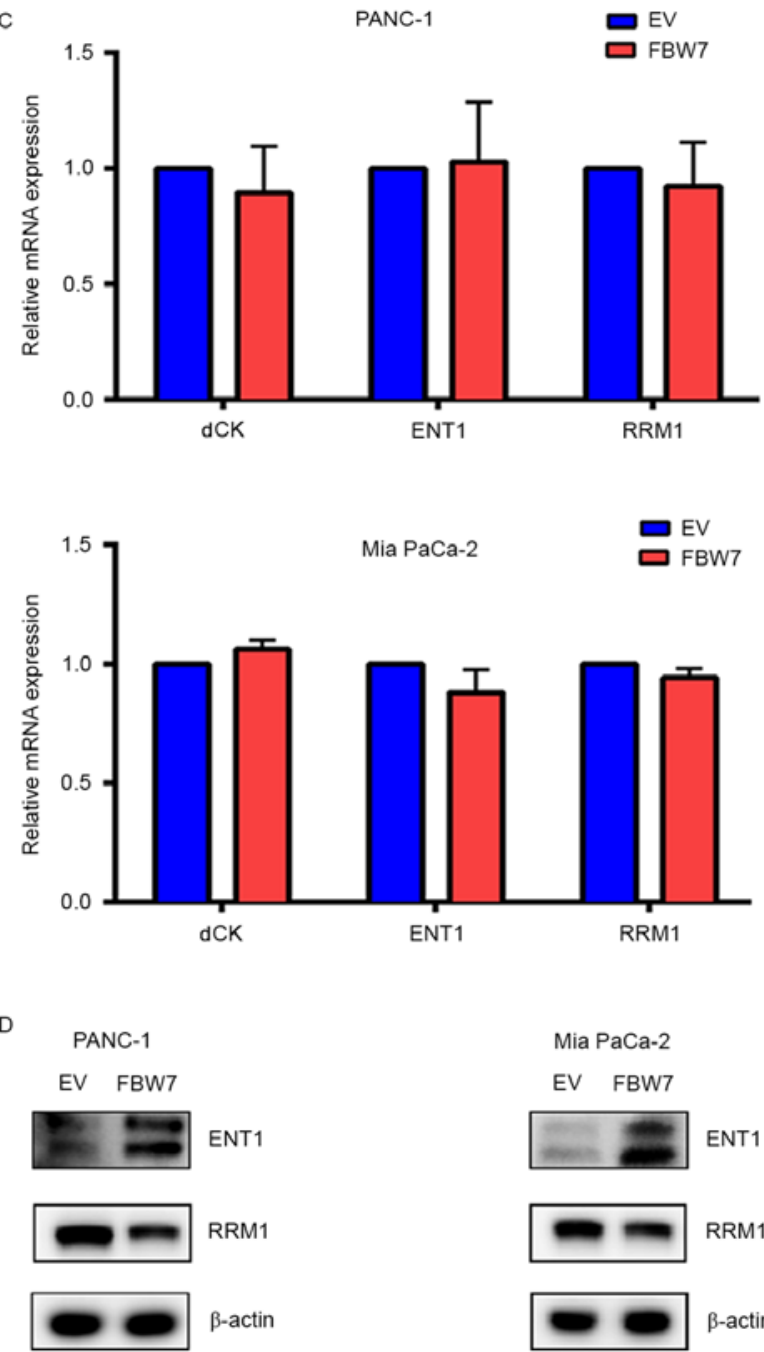

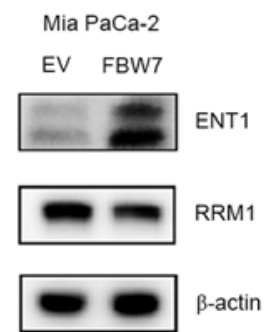

Figure 2. FBW7 regulates the ENT1 protein level. (A) Overexpression of the FBW7 substrate, anti-apoptotic factor MCL-1, is commonly regarded as a factor that regulates drug resistance. However, in the FBW7-overexpressing PANC-1 and Mia PaCa-2 cells, we detected no significant change in MCL-1 level, indicating that other molecular mechanisms underlie gemcitabine resistance. (B) Schematic representation of gemcitabine activation. ENT1 is responsible for gemcitabine transport, dCK catalyzes the phosphorylation of gemcitabine for subsequent activation, and RRM1 is inhibited by activated gemcitabine. ENT1, dCK and RRM1 are indicators of gemcitabine efficacy in pancreatic cancer. (C) FBW7 did not participate in the regulation of ENT1, dCK and RRM1 at the transcriptional level. (D) FBW7 increased the protein levels of ENT1 in PANC-1 and Mia PaCa-2 cells, while it had slight influence on the RRM1 protein level. dCK was not detected in the present study. GEM, gemcitabine.

an increase in the ENT1 level in the cell membrane (Fig. 4C). These results suggest that ERK kinase activity may be responsible for the decrease in ENT1, which subsequently renders resistance to chemotherapy. Moreover, the ERK/FBW7 axis may function as a novel target for improving chemotherapy sensitivity.

\section{Discussion}

Pancreatic cancer is a lethal disease, and its death rate is almost equal to its incidence. Despite significant progress in the diagnosis and treatment of the disease, its 5-year survival rate remains desperately low at $\sim 6 \%$. Surgical resection, chemotherapy and radiotherapy are the primary options for the treatment of pancreatic cancer. However, due to early metastasis, only a small proportion of patients are suitable for surgery. Thus, gemcitabine-based chemotherapy has a pivotal role in the treatment of locally advanced and metastatic pancreatic cancer. Yet, the response rate is still unsatisfactory and gemcitabine resistance remains a hurdle for the improvement of overall survival. Thus, understanding the biological mechanisms involved in gemcitabine resistance may help to improve the efficacy of gemcitabine-based chemotherapy (18).

Our previous studies uncovered novel functions of FBW7 as a novel tumor-suppressor in pancreatic cancer via suppressing c-Myc, and c-Myc-induced malignancies such as aerobic glycolysis, increased CA125 production and the resultant metastasis (11-13). However, its role in the resistance to chemotherapy in pancreatic cancer has seldom been discussed. In the present study, we analyzed the contribution of FBW7 in gemcitabine resistance and observed that introduction of FBW7 renders sensitivity to gemcitabine in PANC-1 and Mia PaCa-2 cells. To uncover the underlying molecular mechanism, we firstly examined the level of anti-apoptotic factor MCL-1 in FBW7-overexpressing PANC-1 and Mia PaCa-2 cells. MCL-1 is a FBW7 substrate and possesses anti-apoptotic 
A

$$
\text { PANC-1 }
$$

EV FBW7

E 3 ENT1

- TFR

$-\infty$ APTlAl
Mia PaCa-2

EV FBW7

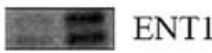

- TFR

B
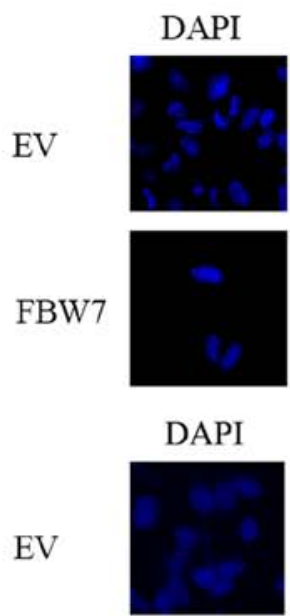

FBW7
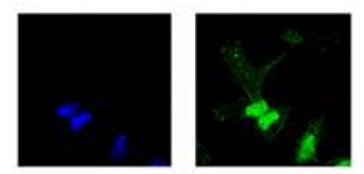

Mia PaCa-2
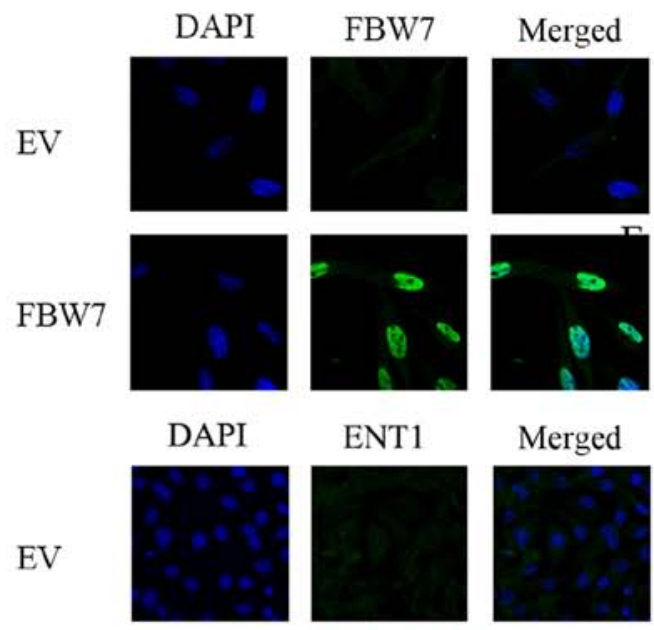

FBW7
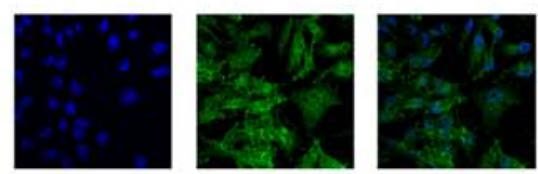

C

Low
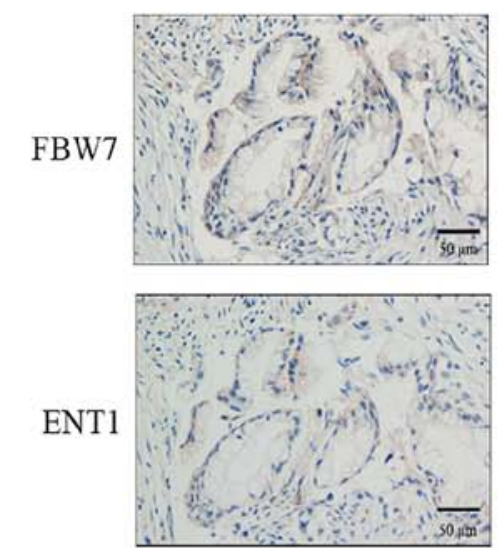

D

PANC-1

Ctrl MG132

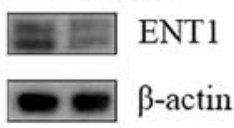

E

PANC-1

Mia PaCa-2

Ctrl CQ

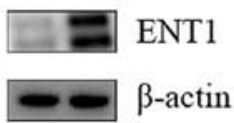

Ctrl CQ

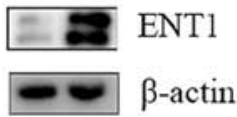

F

PANC-1

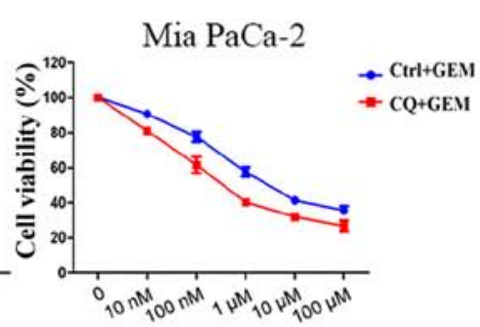

G

PANC-1

Mia PaCa-2

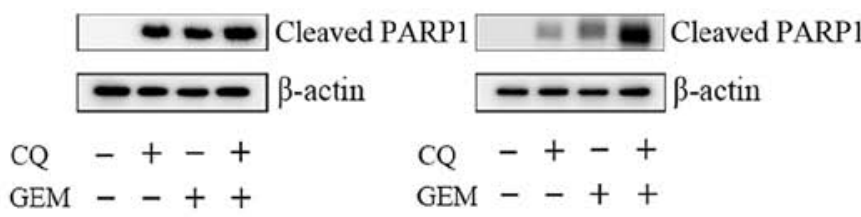

Figure 3. FBW7 regulates ENT1 membrane distribution. (A) Membrane-bound ENT1 is responsible for efficient gemcitabine uptake. In FBW7-overexpressing PANC-1 and Mia PaCa-2 cells, we detected an increase in the membrane level of ENT1, indicating a positive role of FBW7 in the ENT1 protein level and membrane distribution regulation. (B) Immunofluorescence studies demonstrated that in FBW7-overexpressing PANC-1 and Mia PaCa-2 cells, the membrane localized ENT1 was higher than that in the control empty vector (EV)-transfected cells. (C) IHC staining in pancreatic cancer patient tissues demonstrated that patients with higher FBW7 displayed higher ENT1 expression, further validating the positive correlation between FBW7 and ENT1. (D) Proteosomal inhibitor MG132 did not increase the protein level of ENT1. (E) Pancreatic cancer cells PANC-1 and Mia PaCa-2 were treated with chloroquinone (CQ), a lysosome inhibitor. In addition, upon CQ treatment, the protein level of ENT1 was increased significantly. (F) CQ treatment increased gemcitabine sensitivity in PANC-1 and Mia PaCa-2 cells. (G) CQ treatment increased the level of cleaved PARP1, which also supported the hypothesis that gemcitabine resistance may involve the lysosome degradation of ENT1. GEM, gemcitabine. 
A

$$
\text { PANC-1 }
$$

Ctrl UO126

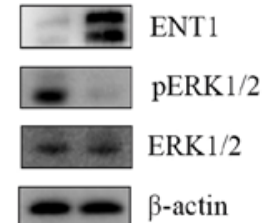

Mia PaCa-2

Ctrl UO126

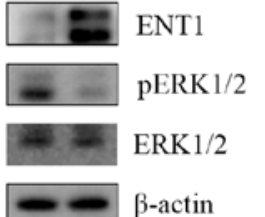

C

PANC-1

Mia PaCa-2

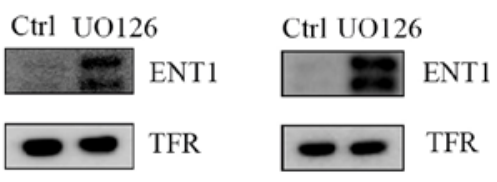

Mia PaCa-2

PANC-1

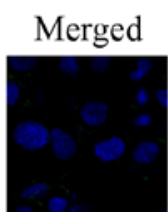

Ctrl
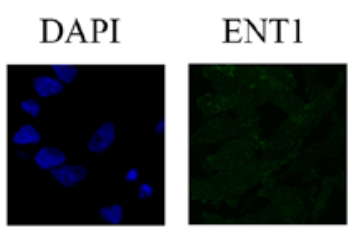

Merged
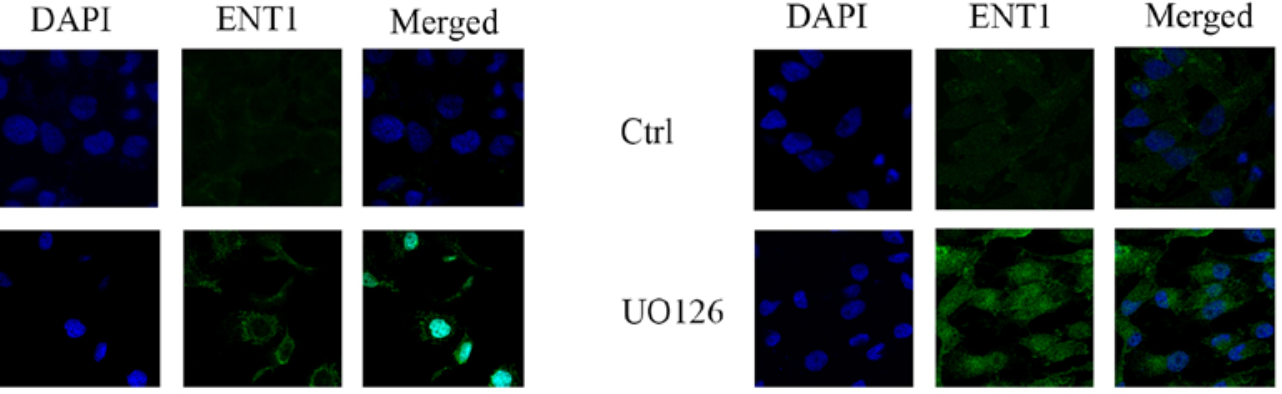

UO126

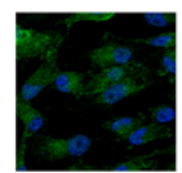

Figure 4. FBW7 upstream regulator ERK regulates ENT1 abundance. (A) PANC-1 and Mia PaCa-2 cells were treated with UO126, which regulated FBW7 protein stability in pancreatic cancer. In addition, upon UO126 treatment, the protein level of ENT1 was increased significantly. (B) Immunofluorescence studies demonstrated that UO126 treatment not only increased total ENT1 level, but also increased cell membrane distribution of ENT1 in PANC-1 and Mia PaCa-2 cells. (C) Membrane purification and western blotting further indicated that UO126 treatment could increase membrane levels of ENT1 in PANC-1 and Mia PaCa-2 cells.

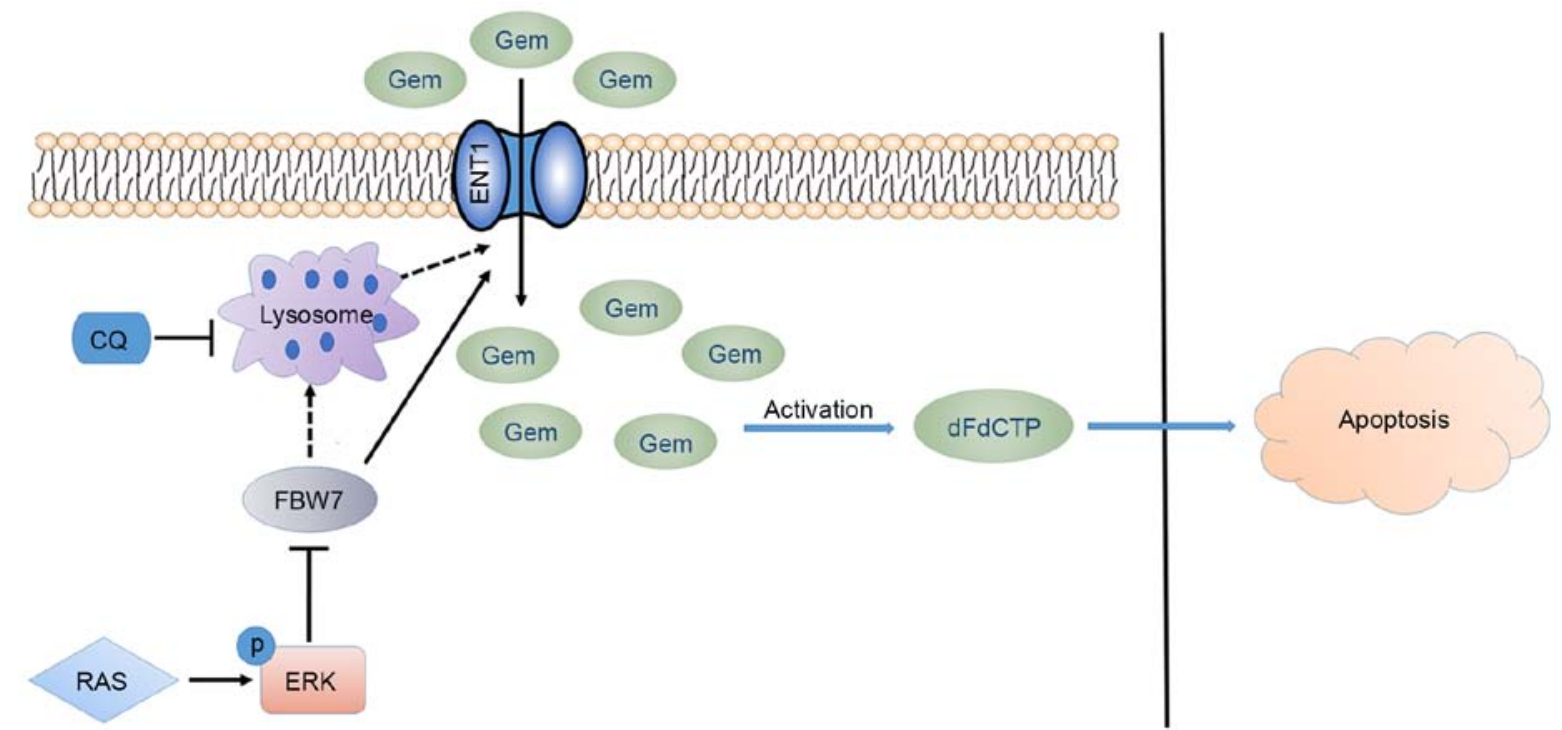

Figure 5. Illustration of the working model. FBW7 overexpression or induction by ERK inhibition increased gemcitabine sensitivity by induction of gemcitabine transporter ENT1. Further studies demonstrated that the protein level of ENT1 was regulated by lysosomes, and inhibition of lysosomes could increase the ENT1 level. Therefore, the FBW7/ENT1 axis may function as a novel therapeutic target in the treatment of pancreatic cancer. GEM, gemcitabine.

functions. Previous studies have demonstrated that MCL1 is overexpressed in many types of cancers, and its upregulation renders cytotoxic resistance (19-21). However, in the present study, we observed no variations in MCL-1 in the FBW7overexpressing cells. Thus, we investigated changes in the expression of three genes associated with gemcitabine uptake and metabolism, including ENT1, dCK and RRM1. Decreased expression of these genes was found to predict worse prognosis of pancreatic cancer patients and are associated with innate and acquired gemcitabine resistance. Our results demonstrated that FBW7 regulated proteins levels of ENT1 but not at the transcriptional level. To ascertain the possible mechanism, we treated cells with MG132, a proteasome inhibitor. The results indicated that MG132 exerted no significant impact on the 
protein levels of ENT1. Lysosomes are dynamic organelles that receive and degrade macromolecules from the secretory, endocytic, autophagic and phagocytic membrane-trafficking pathways (22). The importance of lysosomes in oncogenesis, cancer progression, metastasis and apoptosis have been revealed in recent years (23). Thus, we treated the cells with the lysosome inhibitor, chloroquinone, and observed a significant increase in the protein level of ENT1. These results suggest that lysosome-mediated degradation of ENT1 may play a novel role in the acquisition of gemcitabine resistance. However, the direct molecular mechanisms underlying the membrane endocytosis and lysosome degradation need further investigation. Membrane endocytosis and subsequent autophagosome and lysosome degradation is a multi-step process (24). The membrane intake by vesicular traffic is the first step, and ADP-ribosylation factor (ARF) small GTPases regulate vesicular traffic and organelle structure by recruiting coat proteins to form cargos for intracellular traffic (25). Among these ARF proteins, ARF1 and ARF6, are two of the best characterized ARF proteins that function in biological processes such as secretion, endocytosis, phagocytosis, cell adhesion and tumor cell invasion (26). Membrane proteins that are sorted for intracellular transporting are transported to destinations such as the endoplasmic reticulum (ER), Glogi apparatus, autophagosomes and lysosomes. Recent years have witnessed the importance of membrane protein intracellular trafficking in the progression of cancer cells (27-29). For example, epidermal growth factor receptor (EGFR) intracellular transporting and recycling have been observed in many cancers, promoting uncontrolled proliferation and metastasis of cancer cells $(27,30,31)$. Another example is the activated hepatocyte growth factor (HGF) receptor (Met) which undergoes rapid endocytosis and uniquitin-dependent sorting to the lysosomal degradation pathway $(32,33)$. Recent studies have demonstrated that this mode of downregulation can be circumvented by mutant receptors bearing kinase-activating mutations that instead recycle to the plasma membrane. These mutant receptors can elicit enhanced signaling from endosomes, which is critical for cell motility and tumorigenesis (34). Therefore, strategies to target membrane endocytosis and lysosomal degradation may provide novel therapeutic opportunities for inhibition of malignant behaviors such as proliferation, invasion and metastasis (35-37).

In the present study, we demonstrated that the tumorsuppressor FBW7 promoted sensitivity to gemcitabine in pancreatic cancer cells. Mechanistic studies demonstrated that FBW7 increased the protein levels of gemcitabine transporter ENT1 in PANC-1 and Mia PaCa-2 cells. Further studies demonstrated that lysosomal inhibition by chloroquinone also increased the protein levels of ENT1. These observations indicated the novel functions of FBW7 in chemotherapy resistance and lysosome or autophagosome function (Fig. 5), and uncovered novel aspects for improving drug resistance in pancreatic cancer.

\section{Acknowledgements}

The present study was supported by the National Natural Science Foundation (nos. 81372651 and 81502031), the Sino-German Center (GZ857), Ph.D. Programs Foundation of Ministry of Education of China (20120071120104), and the Program of Science and Technology Commission of Shanghai (nos. 13431900105 and 13DZ1942802).

\section{References}

1. Vincent A, Herman J, Schulick R, Hruban RH and Goggins M: Pancreatic cancer. Lancet 378: 607-620, 2011.

2. Siegel RL, Miller KD and Jemal A: Cancer statistics, 2015. CA Cancer J Clin 65: 5-29, 2015.

3. Saif MW: Advanced stage pancreatic cancer: Novel therapeutic options. Expert Rev Clin Pharmacol 7: 487-498, 2014.

4. Cid-Arregui A and Juarez V: Perspectives in the treatment of pancreatic adenocarcinoma. World J Gastroenterol 21: 9297-9316, 2015.

5. Conroy T, Desseigne F, Ychou M, Bouché O, Guimbaud R, Bécouarn Y, Adenis A, Raoul JL, Gourgou-Bourgade S, de la Fouchardière C, et al; Groupe Tumeurs Digestives of Unicancer; PRODIGE Intergroup: FOLFIRINOX versus gemcitabine for metastatic pancreatic cancer. N Engl J Med 364: 1817-1825, 2011.

6. Von Hoff DD, Ervin T, Arena FP, Chiorean EG, Infante J, Moore M, Seay T, Tjulandin SA, Ma WW, Saleh MN, et al: Increased survival in pancreatic cancer with nab-paclitaxel plus gemcitabine. N Engl J Med 369: 1691-1703, 2013.

7. Wang Z, Inuzuka H, Zhong J, Wan L, Fukushima H, Sarkar FH and Wei W: Tumor suppressor functions of FBW7 in cancer development and progression. FEBS Lett 586: 1409-1418, 2012.

8. Min SH, Lau AW, Lee TH, Inuzuka H, Wei S, Huang P, Shaik S, Lee DY, Finn G, Balastik M, et al: Negative regulation of the stability and tumor suppressor function of Fbw7 by the Pin1 prolyl isomerase. Mol Cell 46: 771-783, 2012.

9. Akhoondi S, Sun D, von der Lehr N, Apostolidou S, Klotz K, Maljukova A, Cepeda D, Fiegl H, Dafou D, Marth C, et al: $F B X W 7 / h C D C 4$ is a general tumor suppressor in human cancer. Cancer Res 67: 9006-9012, 2007.

10. Akhoondi S, Lindström L, Widschwendter M, Corcoran M, Bergh J, Spruck C, Grandér D and Sangfelt O: Inactivation of $F B X W 7 / h C D C 4-\beta$ expression by promoter hypermethylation is associated with favorable prognosis in primary breast cancer. Breast Cancer Res 12: R105, 2010.

11. Ji S, Qin Y, Shi S, Liu X, Hu H, Zhou H, Gao J, Zhang B, Xu W, Liu J, et al: ERK kinase phosphorylates and destabilizes the tumor suppressor FBW7 in pancreatic cancer. Cell Res 25: $561-573,2015$.

12. Ji S, Qin Y, Liang C, Huang R, Shi S, Liu J, Jin K, Liang D, $\mathrm{Xu}$ W, Zhang B, et al: FBW7 (F-box and WD repeat domaincontaining 7) negatively regulates glucose metabolism by targeting the c-Myc/TXNIP (thioredoxin-binding protein) axis in pancreatic cancer. Clin Cancer Res 22: 3950-3960, 2016.

13. Liang C, Qin Y, Zhang B, Ji S, Shi S, Xu W, Liu J, Xiang J, Liang D, Hu Q, et al: Oncogenic KRAS targets MUC16/CA125 in pancreatic ductal adenocarcinoma. Mol Cancer Res 15: 201-212, 2017.

14. Maréchal R, Bachet JB, Mackey JR, Dalban C, Demetter P, Graham K, Couvelard A, Svrcek M, Bardier-Dupas A, Hammel P, et al: Levels of gemcitabine transport and metabolism proteins predict survival times of patients treated with gemcitabine for pancreatic adenocarcinoma. Gastroenterology 143: 664-674, 2012.

15. Nordh S, Ansari D and Andersson R: hENT1 expression is predictive of gemcitabine outcome in pancreatic cancer: A systematic review. World J Gastroenterol 20: 8482-8490, 2014.

16. Spratlin J, Sangha R, Glubrecht D, Dabbagh L, Young JD, Dumontet C, Cass C, Lai R and Mackey JR: The absence of human equilibrative nucleoside transporter 1 is associated with reduced survival in patients with gemcitabine-treated pancreas adenocarcinoma. Clin Cancer Res 10: 6956-6961, 2004.

17. Plunkett W, Huang P, Searcy CE and Gandhi V: Gemcitabine: Preclinical pharmacology and mechanisms of action. Semin Oncol 23 (Suppl 10): S3-S15, 1996.

18. Kourie HR, Gharios J, Elkarak F, Antoun J and Ghosn M: Is metastatic pancreatic cancer an untargetable malignancy? World J Gastrointest Oncol 8: 297-304, 2016.

19. Ertel F, Nguyen M, Roulston A and Shore GC: Programming cancer cells for high expression levels of Mcl1. EMBO Rep 14: 328-336, 2013. 
20. Wertz IE, Kusam S, Lam C, Okamoto T, Sandoval W, Anderson DJ, Helgason E, Ernst JA, Eby M, Liu J, et al: Sensitivity to antitubulin chemotherapeutics is regulated by MCL1 and FBW7. Nature 471: 110-114, 2011.

21. Lucchetti C, Rizzolio F, Castronovo M and Toffoli G: Research highlights. MCL1 and FBW7 as new predictive candidate biomarkers of anti-tubulin agents. Pharmacogenomics 12: $1379-1380,2011$.

22. Kirkegaard $\mathrm{T}$ and Jäättelä M: Lysosomal involvement in cell death and cancer. Biochim Biophys Acta 1793: 746-754, 2009.

23. Guicciardi ME, Leist M and Gores GJ: Lysosomes in cell death. Oncogene 23: 2881-2890, 2004.

24. Maxfield FR and McGraw TE: Endocytic recycling. Nat Rev Mol Cell Biol 5: 121-132, 2004.

25. Seixas E, Barros M, Seabra MC and Barral DC: Rab and Arf proteins in genetic diseases. Traffic 14: 871-885, 2013

26. D'Souza-Schorey $C$ and Chavrier P: ARF proteins: Roles in membrane traffic and beyond. Nat Rev Mol Cell Biol 7: 347-358, 2006.

27. Le Roy C and Wrana JL: Clathrin- and non-clathrin-mediated endocytic regulation of cell signalling. Nat Rev Mol Cell Biol 6: 112-126, 2005.

28. Rainero E: Extracellular matrix endocytosis in controlling matrix turnover and beyond: Emerging roles in cancer. Biochem Soc Trans 44: 1347-1354, 2016.
29. White E, Mehnert JM and Chan CS: Autophagy, metabolism, and cancer. Clin Cancer Res 21: 5037-5046, 2015.

30. Peng K, Dai Q, Wei J, Shao G, Sun A, Yang W and Lin Q: Stress-induced endocytosis and degradation of epidermal growth factor receptor are two independent processes. Cancer Cell Int 16: 25, 2016.

31. Sorkin A: Internalization of the epidermal growth factor receptor: Role in signalling. Biochem Soc Trans 29: 480-484, 2001

32. Joffre C, Barrow R, Ménard L, Calleja V, Hart IR and Kermorgant S: A direct role for Met endocytosis in tumorigenesis. Nat Cell Biol 13: 827-837, 2011.

33. Seton-Rogers S: Signalling: Location, location, location. Nat Rev Cancer 11: 462-463, 2011

34. Clague MJ: Met receptor: A moving target. Sci Signal 4: pe40, 2011.

35. Mosesson Y, Mills GB and Yarden Y: Derailed endocytosis: An emerging feature of cancer. Nat Rev Cancer 8: 835-850, 2008.

36. Tardy C,CodognoP, Autefage H,Levade T and Andrieu-Abadie N: Lysosomes and lysosomal proteins in cancer cell death (new players of an old struggle). Biochim Biophys Acta 1765: 101-125, 2006.

37. Appelqvist $\mathrm{H}$, Wäster $\mathrm{P}, \mathrm{K}$ ågedal $\mathrm{K}$ and Öllinger $\mathrm{K}$ : The lysosome: From waste bag to potential therapeutic target. J Mol Cell Biol 5: 214-226, 2013. 